\section{THE FIELD LINE TOPOLOGY OF A UNIFORM MAGNETIC FIELD SUPERPOSED ON THE FIELD OF A DISTRIBUTED RING CURRENT*}

\author{
M.S. CHANCE \\ Plasma Physics Laboratory, Princeton University \\ P.O. Box 451, Princeton, New Jersey 08543 \\ DE91 015377
}

PPPL --2767

\title{
J.M. GREENE and T.H. JENSEN
}

General Atomics, P.O. Box 85608.

Sar: Diego, California 92186-9784

\begin{abstract}
A magnetic field line topology with nulls, generated by superimposing a uniform magnetic field onto the field from a distributed ring current, is analyzed. This simple model is amenable to substantial analytical progress and aiso facilitates the visualization of the three dimensional field geometry. Four nulls are seen to exist and representative field iines and tubes of flux found by numerical integration are presented. An infinite number of topologically distinct flux bundles is found. A convenient mapping is defined which proves very useful in distinguishing between and following the paths of the different tubes of flux as they traverse through the null system. The complexities already present in this simple but nontrivial configuration serve to emphasize the difficulties in analyzing more complicated geornetries, but the intuition gained from this study proves beneficial in those cases. One such example is the application to a model of plasmoid formations in the earth's magnetotail.
\end{abstract}

\section{INTRODUCTION}

The appearance of nulls in the magnetic field topology of a magnetized plasma can drastically alter its dynamic processes. Since these nulls are arguably the most essential ingredient in three dimensional magnetic reconnection processes these are, no doubt, largely responsible for many of the rich variety of phenomena observed in space, solar, or astrophysical plasmas. For example, such nulls can appear through the dynamic interaction of the interplanetary magnetic field (IMF) with the dipolar field of the earth, and in a sense, open up the hitherto closed field lines to the full onslaught of the solar wind plasma. The ensuing plasma activity is generally assumed to be the cause of many of the earthbound or satellite measurements in space. The study of the topology of magnetic fields is in itself an important and fascinating

* This work was supported in part by the U.S. Department of Energy Contract No. DE-AC0276-CHO-3073, and NASA Headquarters Contract No. NASW-4393. 
subject and overlaps intimately with the studies of chaotic behavior in nonlinear dynamics - the research in these areas in the field of nuclear fusion can attest to this. Knowledge of such magnetic field topology is essential since it provides the fundamental framework upon which the plasma-physical processes take place, but, because of the three dimensional nature of the problem, it is difficult to obtain a compreher:ive picture of even the most simple non-trivial case.

This paper presents the results of a detailed study of the field line topology of a simple but nontrivial configuration comprised of a constant magnetic field superposed on the field of a distributed ring current. This simple case contains most of the essential ingredients necessary for our study and greatly enhances our coinprenension of the topology since the analysis is simplified and the details can be more lucidly presented. The paper provides another demonstration of the thesis that magnetic nulls are necessary in order for a typical three-dimensional configuration to possess a variety of topologically distinct field lines. In the present case, the topological distinction between field lines is the number of times they loop around a circle that is itself composed of field lines. As shown in Appendix A, null points lie on the circle. Further, the boundaries between the different flux tubes are formed of field lines that join to nulls. The techniques used and the knowledge gained in this model should be applicable to more realistic and more complex situations. We demonstrate such an application in a less detailed study on a more physical model taken from the earth's magnetotail. In this case the field lines encircle instead a more complicated spiralling field line. It should be apparent also that the results of this study are not solely restricted to magnetic fields but may be directly applicable, for example, to the flow of an incompressible fluid impinging upon vortex generating barriers.

We shall rely substantially on numerical computations and graphics, and as an aid to visualizing the magnetic field lines in the inherently three dimensional geometry of our configuration some of the results are presented in the form of stereographic pairs of figures. These are to be viewed in the appropriate manner, j.e., relaxing the eyes as if looking far beyond the figures so that the images from each eye merge into a single unit, while at the same time trying to focus on the pictures. The ihree dimensional impact should be readily apparent if done correctly. Inserting a card or similar object between and perpendicular to the figures may prove to be helpiul. Alternatively, 
it may be easier to view the figures by crossing the eyes instead, in which case, the perspective will be reversed and somewhat distorted but the essential topological features will still be discernible. The easiest way is to view them, preferably enlarged, through a stereoscope designed for this purpose.

Previous noteworthy papers have addressed the physical consequences of magnetic nulls, and also the mathematical analysis of the behavior of the magnetic field in the neighborhood of the nulis (Cowley, 1973; Stern, 1973, 1979; Fukao, et al., 1975; Greene, 1988). To avoid redundancy, only minimal emphasis will be placed on these topics in this work.

In the next section we describe our model of the magnetic configuration of the ring current case together with its symmetry properties. Four nulls are shown to exist. In Section 3 we briefly describe the eigenanalysis of the magnetic field lines near the null points and apply it to our dipole-IMF configuration. The section following shows the result of numerically integrating the field line equations for the global structure of the magnetic field. The structure is quite complex and still difficult to visualize completely and quantitatively even when viewed stereoscopically, but a mapping of the lines is described in Section 5 in which the complicated flux bundles are sorted out systematically by identifying their various $X$-points and separatrices and hence serves to clarify the topology of the configuration. Section 6 contains a comparison of our study with that of the topology of a configuration analyzed by Birn, et al. (1989) which was modeled after the plasmoid formation in the earth's magnetotail. In Section 7, concluding remarks are presented. Finally, in order not to have diverted attention from the main text, we defer to Appendix $A$ a discussion of the coalescence curves whereupon pairs of nulls in our distributed current ring model can merge, and to Appendix B a derivation for general conditions for the presence of magnetic field lines which lie on circles and connect the null pairs of the same configuration.

\section{DESCRIPTION OF THE RING CURRENT MODEL}

The model chosen here is that of a uniform magnetic field superposed on the magnetic field arising from a finite and diffuse current ring. This can be thought of as representative of the interaction of the earth's dipole field with the interplanetary magnetic field in which it is immersed. This study was in fact motivated by the 
point dipole-IMF analyses of Cowley (1973), and Stern (1973). The analogy is quite ideclized since we ignore any topological changes caused by the presence of the earth. We choose here a current ring of finite radius as opposed to a point dipole since the latter possesses an inherent singularity at the dipole itself. This tends to complicate the analysis and also masks the behavior of the magnetic field in the neighborhood of the dipole, where, as we shall see, important topological contributions are found. For somewhat the same reasons we choose a diffuse current ring, and one that can be described algebraically, thus avoiding the transcendental and singular nature of the elliptic integrals associated with a filamentary current loop. We leave aside the question of force balance in our configuration under the assumption that the general topology will not be drastically altered under the constraints of an equilibrium state. This remains to be shown rigorously.

The notation used for the cartesian, cylindrical, and spherical coordinate systems used in this paper is such that $(x, y, z)=(\rho, \phi, z)=(r, \theta, \phi)$, respectively. Thus the vector potential chosen for the dipolar-like magnetic field $B$, is given, in arbitrary units, by

$$
A_{0}=-\rho / R^{3} \text {, }
$$

with the associated current ring

$$
j_{\phi}=-15 a^{2} \rho / R^{7}
$$

and magnetic field components

$$
\begin{aligned}
B & =-\left(3 x z, 3 y z, 2 z^{2}-x^{2}-y^{2}+2 a^{2}\right) / R^{5} \\
& =-\left(3 \rho z, 0,2 z^{2}-\rho^{2}+2 a^{2}\right) / R^{5} \\
& =-\left[2 R^{2} \cos \theta,\left(r^{2}-2 a^{2}\right) \sin \theta, 0\right] / R^{5}
\end{aligned}
$$

in cartesian, cylindrical, and spherical coordinates respectively. Here,

$$
R^{2}=x^{2}+y^{2}+z^{2}+a^{2}
$$

and henceforth, wher not explicitly shown we shall set $a=1$.

Because of the azimuthal symmetry of the dipolar field we can define its poloidal magnetic dipole flux, $\psi$, where

$$
\psi=-2 \pi \rho A_{\phi}=2 \pi \rho^{2} / R^{3},
$$


so that

$$
\boldsymbol{B}=(\nabla \phi \times \nabla \psi) / 2 \pi
$$

Contours of $\psi$ will therefore coincide with the magnetic field lines for the dipolar field. These are shown in Figure 1. Somewhat more quantitative information can be obtained from the level curves for the orientation angle, $\omega$, and the magnitude of the dipolar field (or, alternatively, $B_{z}$ and $B_{\rho}$ ). These are superimposed in Figure 2. The dotted curves represent equal increments in $\omega$ whereas for clarity the solid contour lines for $|B|$ are equally spaced along the diagonal of the figure. Because of symmetry it is necessary to show only the first quadrant. The symmetry is such that $\boldsymbol{B}(\boldsymbol{r})=\boldsymbol{B}(-\boldsymbol{r})$ so that the magnetic field in the first(second) quadrant is the reflection of that in the third(fourth) quadrant. The uniform IMF shares this symmetry, so that it is also satisfied for the total field, $\boldsymbol{B}_{t}$, in the present configuration. Thus nulls created by a superimposed IMF which is constant in magnitude and direction will occur in pairs at $\boldsymbol{r}$ and $-\boldsymbol{r}$. Some quantitative features to note about Figure 2 are the $O$ points at $(\rho, z)=(0,0)$ and $\left(2^{1 / 2}, 0\right)$, and the saddle point at $(2,0)$ in the contour lines for $|\boldsymbol{B}|$. It can also be seen from the figure that generally two pairs of aulls will be present since a particular curve of constant $\omega$ will intersect a curve of constant magnitude twice in the same quadrant. This may be thought to be an artifact of the diffuse current model which is chosen since the dipolar component of the magnetic field vanishes at the two-dimensional $O$-point at $\rho=2^{1 / 2}$, and thus must increase to a maximum before decaying away again at large $r$. Note, however, that this vanishing will occur in any current filament of finite thickness.

The pair of nulls in a given quadrant can coalesce along the locus where the two sets of level curves in Figure 2 osculate. The equation for this locus shown as the dashed line, is derived in Appendix A. It separates the two classes of nulls. The ones farther from the curreat channel will be called the primary nulls and the inner ones secondary nulls. Other relevant features will also be discussed in that appendix.

\section{THE EIGENSYSTEM}

For a uniform IMF the eigenanalysis presented in this section is dependent only on the properties of the dipolar field. We shall therefore suppress the subscript $t$ that denotes the total field in what follows. 
The behavior of the magnetic field in the neighborhood of a null point is adequately presented in previous publications (Fukao, 1975; Greene, 1988). In essence, since

$$
d B=\frac{\partial B}{\partial x} d x+\frac{\partial B}{\partial y} d y+\frac{\partial B}{\partial z} d z
$$

then

$$
B \approx B\left(r_{n}\right)+(d r \cdot \nabla) B .
$$

Along principal field lines that end at a null at $r_{n}$ we find the relation

$$
B \approx \lambda d \boldsymbol{r},
$$

so that their configuration is described by the eigensystem,

$$
(d r \cdot \nabla) B-\lambda d r \equiv \overline{\nabla \bar{B}} \cdot d r-\lambda d r=0 .
$$

Since $\nabla \cdot B=0=$ trace $\widetilde{\nabla B}$, the sum of the eigenvalues is zero, so either the magnetic field along one eigenvector points out of the null while it points inward along the other two eigenvectors ( type- $A$ nulls (Cowley, 1973) ), or the converse may occur (type- $B$ nulls). This distinction is preserved if $d r$ is replaced by $-d r$ in (9). The field lines that join to these two tjpes of nulls are depicted in Figure 3 . The eigenvectors shown in the upper sketches are orthogonal. In the circumstance that the eigenvectors are not orthogonal, the figure is distorted but basically unchanged. A sketch of field lines for the case where the $\widetilde{\nabla B}$ matrix has complex eigenvalues is given in the lower half of Figure 3 . The properties of these configurations are more fully discussed by Cowley (1973) and Greene (1988) and the notations used here are generally consistent with the ones used in those papers.

Lines of force that come close to the nulls lie on approximate hyperbolae. They asymptote along lines labeled $\gamma$ at one end, and to planes labeled $\Sigma$ at the other end. It follows that the configuration of the lines of force in the vicinity of a null is essentially that of a two-dimensional $X$-point rotated around the axis labeled $\gamma_{A}$ or $\gamma_{B}$. Thus the magnetic lines that lie along these axes will be called spindle lines. The surfaces denoted by $\Sigma_{A}, \Sigma_{B}$, are generated by linear combinations of the four ingoing or outgoing field lines around the $A$ and $B$ nulls respectively. The eigenvalues of $\widetilde{\nabla B}$ can be complex, in which case the feld lines spiral into $A$ nulls, or out of $B$ nulis. 
Such configurations are also illustrated in figure 3. These complex eigenvalues play an important role in the configuration studied in this paper. A minimum current flowing through the null is required in order to obtain complex eigenvalues and to provide the net circulation for spiralling to occur, since in the absence of current $\widetilde{\nabla B}$ is symmetric and so has real eigenvalues.

For cases with azimuthal symmetry and where $B_{\phi}=0$ the $\widetilde{\nabla B}$ matrix is block diagonalized and the eigenequations are given by $\Lambda \cdot V=0$, where

$I$ being the unit dyadic.

$$
\Lambda=\left(\begin{array}{ccc}
\frac{\partial B_{\rho}}{\partial \rho} & 0 & \frac{\partial B_{\rho}}{\partial z} \\
0 & \frac{B_{\rho}}{\rho} & 0 \\
\frac{\partial B_{z}}{\partial \rho} & 0 & \frac{\partial B_{z}}{\partial z}
\end{array}\right)-\lambda \boldsymbol{I},
$$

The eigenvalues are therefore

$$
\begin{aligned}
\lambda_{1} \equiv \lambda_{\phi} & =\frac{B_{\rho}}{\rho} \\
\lambda_{2} & =-\frac{\lambda_{1}}{2}+\frac{\Delta}{2} \\
\lambda_{3} & =-\frac{\lambda_{1}}{2}-\frac{\Delta}{2} .
\end{aligned}
$$

with corresponding unit eigenvectors,

$$
\begin{aligned}
& V_{1}=\hat{e}_{\phi} \\
& V_{ \pm}=\frac{\frac{\partial B_{\rho}}{\partial z} \hat{e}_{p}+\frac{\delta \pm \Delta}{2} \hat{e}_{z}}{\left|\frac{\partial B_{p}}{\partial z} \hat{e}_{\rho}+\frac{\delta \pm \Delta}{2} \hat{e}_{x}\right|}
\end{aligned}
$$

where

$$
\delta=\frac{\partial B_{z}}{\partial z}-\frac{\partial B_{\rho}}{\partial \rho}
$$

and

$$
\begin{aligned}
\Delta & =\left[\lambda_{\phi}^{2}-4 \tilde{D}\right]^{1 / 2} \\
& =\left[\delta^{2}+4 \frac{\partial B_{\rho}}{\partial z} \frac{\partial B_{z}}{\partial \rho}\right]^{1 / 2} \\
& =\left[\delta^{2}+4\left(\frac{\partial B_{z}}{\partial \rho}\right)^{2}-4 j_{\phi} \frac{\partial B_{z}}{\partial \rho}\right]^{1 / 2},
\end{aligned}
$$


where

$$
\tilde{D}=\frac{\partial B_{\rho}}{\partial \rho} \frac{\partial B_{x}}{\partial z}-\frac{\partial B_{\rho}}{\partial z} \frac{\partial B_{x}}{\partial \rho} .
$$

Equation (17) shows explicitly that a large enough current can give rise to complex eigensolutions. Also, it is shown in Appendix A that the equation given by $\bar{D}=0$, is the curve which separates the primary and secondary nulls and therefore it is seen from (15) that the primary nulls always have real eigenvalues.

For our model, the elements of the eigenmatrix are given by

$$
\widetilde{\nabla B}=\frac{3}{\bar{R}^{\bar{T}}}\left(\begin{array}{ccc}
-z\left(R^{2}-5 \rho^{2}\right) & 0 & -\rho\left(R^{2}-5 z^{2}\right) \\
0 & -z R^{2} & 0 \\
\rho\left(4 R^{2}-5 \rho^{2}\right) . & 0 & z\left(2 R^{2}-5 \rho^{2}\right)
\end{array}\right)
$$

If we now superimpose the constant IMF onto the dipolar field we find that it is convenient for the quantitative analysis to choose the locations of the null points of our configuration rather than the value of the MF. The magnetic field at the nulls can then be found directly from (3). The topological properties of the degenerate cases where the IMF points along the $\pm z$ directions are trivial although the topology in one case is distinguished from the more general case by having the field vanish along a line rather than a point. As an example of the more general case, we choose the IMF such that the primary null occurs at $(x, y, z)=(0,1,2)$ in the first quadrant. There the magnetic field of the IMF is found to be

$$
\left(b_{x}, b_{y}, b_{z}\right)=(0.0,0.06804,0.10200)=\left(0.1226,56.29^{\circ}\right)
$$

the latter pair being the magnitude and orientation of the field with the $y$-axis in the $y-z$ plane. The dipole field is equal and oppositely directed at the null point. The secondary null in the same quadrant is found numerically to be at

$$
(x, y, z)=(0.0,1.1187,0.15827)
$$

The primary null is of type $A$ with eigensolutions

$$
\begin{array}{ll}
\lambda_{1}^{A}=-0.06804, & V_{1}^{A}=\hat{e}_{x} \\
\lambda_{2}^{A}=+0.13702, & V_{2}^{A}=\hat{e} \exp \left(i 61.85^{\circ}\right) \\
\lambda_{3}^{A}=-0.06898, & V_{3}^{A}=\hat{e} \exp \left(-i 35.98^{\circ}\right) .
\end{array}
$$


The other member of the primary pair is of type $B$ with eigensolutions

$$
\begin{array}{ll}
\lambda_{1}^{B}=+0.06804, & V_{1}^{B}=\hat{e}_{x} \\
\lambda_{2}^{B}=+0.06898, & V_{2}^{B}=\hat{e} \exp \left(i 144.0^{\circ}\right) \\
\lambda_{3}^{B}=-0.13702, & V_{3}^{B}=\hat{e} \exp \left(-i 118.1^{\circ}\right),
\end{array}
$$

where we have used a polar representation of the real eigenvectors in the $y-z$ plane such that the angles in (20) and (21) are measured from the $y$-axis. The spindle lines $\gamma_{A}, \gamma_{B}$ points along the solutions $V_{2}^{A}, V_{3}^{B}$ respectively. The actual $B$ field in the vicinity of the null is given by Equation (8). The secondary null pair each has one real and two complex eigensolutions since these occur deep within the current channel. The real eigenvectors point along the $x$-direction.

\section{THE GLOBAL MAGNETIC FIELD STRUCTURE}

The equations for the magnetic field lines are given by

$$
\begin{aligned}
& \frac{d \tau}{d l}=\frac{B_{z}}{\left|B_{t}\right|} \\
& \frac{d y}{d l}=\frac{B_{y}+b_{y}}{\left|B_{t}\right|}, \\
& \frac{d z}{d l}=\frac{B_{z}+b_{z}}{\left|B_{t}\right|}
\end{aligned}
$$

where $l$ is the distance along the field lines, and $B_{\mathfrak{z}}=B+b$, the total field. In this form the equations remain finite as the null points are approached. Another feature to note is that since $B_{x}=0$ at $x=0$ in our model, field lines cannot leave or enter the $x=0$ plane and therefore cannot cross it except through a null - where they vanish. This plane divides the space into two topologically independent hemispheres with reflective symmetry. A numerical algorithm, SARTRE, developed to integrate the set of equations (22), also constructs stereoscopic pairs of figures so as to facilitate the visualization of the field lines in three dimensions. These are to be viewed as suggested in the introduction. Should tinis prove cumbersome, the mapping technique described in the next section provides another means to visualize the fields.

We first show in Figure 4 the field line configuration in the $x=0$ plane. These represent true field lines since, as mentioned above, they can never leave or enter this plane except through a null point. In particular, all the null points lie in this plane 
and are located as described in the previous section. The primary $A$ and $B$ nulls are labeled, while the secondary nulls lie at the center of the spirals. This figure if reflected about the $z$-axis, and oriented appropriately, is to be compared with that of Figure 9 in Cowley's paper (1973). They are physically the same except that here we show important details about the field lines deep in the current channel. Had we chosen our primary null much farther away from the origin we would approximate his point-like dipole configuration. The interplanetary lines are identical to those of Cowley's since these are necessarily farther away from the influence of the specifics of the dipolar model chosen. The lines labeled $\Sigma_{A 1}, \gamma_{A 2}, \Sigma_{B 1}$, and $\gamma_{B 2}$ bound a pathway for flux that passes through the center of the configuration. This correspones to open field lines in Cowley's model. The flux that lies between $\gamma_{A 1}$ and $\gamma_{A 2}$, and between $\gamma_{B 1}$ and $\gamma_{B 2}$ converges on, or diverges from, the secondary $A$ and $B$ nulls respectively. These become the counterpart of Cowley's closed field lines. The notion that the field lines are "closed" here is in the sense that the ends are attached to the earth and not closed on themselves. In fact, we shall see that none of our lines close on themselves unless they connect to nulls or join at infinity.

We now mentally visualize the three-dimensional extension of Figure 4 in the neighborhood of the $x=0$ plane. The sheets $\Sigma_{A}^{\prime}$ and $\Sigma_{B}^{\prime}$ of outgoing and ingoing lines at the secondary nulls lie in the $x=0$ plane, so the eigenvectors normal to this plane define spindle lines, $\gamma_{A 1}^{\prime}$ and $\gamma_{B 1}^{\prime}$. It will be seen below that in fact, because of the symmetry of the configuration, these lines connect and form what shall be cailed the secondary null-null line. Inspection of the eigenvectors shows that at the primary nulls, the lines labeled $\gamma$ are spindle lines, and those labeled with $\Sigma$ form part of the sheets of lines. It is thus apparent that the lines labeled $\Sigma_{A 2}$ and $\Sigma_{B 2}$ in Figure 4 are cuts through scrolls that spiral around the secondary null-null line, and terminate on the primary nulls. This picture will be amplified in the next paragraphs where we show the results of integrating the field line equations in three dimensions.

Turning now to the actual visualization of the three-dimensional structure of the field lines we first show those lines converging onto the eigenvectors of the $A$ null at $(0,1,2)$ and the $B$ null at $(0,-1,-2)$ in the stereoscopic pair of Figure 5. Already one can see that the original dipolar field is topologically modified. The spindle lines, $\gamma_{A}$, emanate on either side of the $A$ null and eventually get swept away 
in the distant IMF. The lines associated with the eigenvector in the $x$-direction form a pair of null-null lipes which join onto the $B$ null. Another null-null line joins the third eigenvector to the spiralling eigenvector of the secondary null near the current channel. Note that the counterpart of this field line in the negative $d r$ direction also comes up from the distant IMF. Similar considerations hold for the null at $(0,-1,-2)$ but with due respect for the symmetry of the configuration. Not shown in the figure are the secondary null-null semicircles mentioned above. That these and also the null-null lines joining the primary $A$ and $B$ nulls are semi-circles is a consequence of our chosen model being a member of a particular class of dipolar fields. The analysis showing this is described in Appendix B.

We now trace the field lines along the $\Sigma_{A}$ surface about the primary $A$ null at $(0,1,2)$. We originate these in rays from a small disc in $\Sigma_{A}$ centered around the spindle line $\gamma_{A}$. The null-null lines connecting the primary nulls divide this disc into two halves. The field lines originating from the upper half of our disc are shown in Figure 6a. These all map into the interplanetary field without approaching the secondary nulls in the current channel and will be labeled $\Sigma_{A 1}$. Those magnetic lines originating from the lower half are shown in Figure $6 \mathrm{~b}$ and will be labeled $\Sigma_{A 2}$. They are seen to spiral away from the $A$-null at $(0,1,2)$, around the secondary null-null circle, then emerge into the IMF from the vicinity of the primary- $B$ null at $(0,-1,-2)$. A close inspection of the figure shows that the number of times that a feld line spirals around varies from $\infty$ for the one which encircles the secondary null in the $x=0$ plane, to 1 for those near the primary null-null circle. The spirals are tighter for those lines emerging from near the $x=0$ plane. A more densely packed sample of lines would include those which actually connect to the primary $B$-null after encircling the secondary null-null circle a countable number of times. An example of such a line spiralling once around the circle can be seen among the three lines shown in Figure 7.

For completeness we show in Figures $\dot{8}$ and 9 the field lines originating from half discs of larger radii and displaced along the spindle lines, $\gamma_{A}$ somewhat above (Fignre 8) and somewhat below (Figure 9) the null. The relation of these figures to those of Figure 6 should be clear. 


\section{MAPPING ONTO THE SECONDARY NULL-NULL PLANE}

The three-dimensional views of the field lines naturally provide ecsier visualination than two-dimensional projections or verbal descriptions. This is especially true when only a few lines are in consideration since they can be easily traced. When the topology is complicated and a global picturiz is desired one can construct it from many such viewings but a total integrated visualization may still be difficult and perhaps impractical. It would also be more useful to have a better quanticative description of the topology of the system. These shortcomings can be alleviated by constructing 2 . mapping of the lines in the following way.

Since all the field lines, except for the interplaneta-y ones, intertwine tise secondary null-null circle it is convenient to use the cisk bounded by iris circle as a reference plane on which to map and distinguish the ${ }^{1:}$,es as they intersect the plane. This is possible since field lines nevez intersect each other. We therefore construct a 'puncture plot' on this plane by following all tie field lines originating on the plane and counting the number of times they intersect the plane while labeling their positions by the number of iniersections. An example of the 'raw data' of such a plot on a relatively coarse grid is shown in Figure 14 . They are numbered $1,2, \ldots 8,9, a, b, \ldots z(=\infty)$, It looks quite complicated so in orde: to trake sense of the mapping systematically, we cunstruct schematic diagrams baz ed $\sigma / 4$ these raw puncture plots and first show how various segments of the $\Sigma$ surf sces map onto the plane. Four segments are differentiated, each formed from the those parts of $\Sigma_{h_{2}}$ and $\Sigma_{B_{2}}$ on either side of the $x=0$ plane (the lines aljng $\Sigma_{A t}$ and $\Sigma_{B 1}$ do not intersect the plane). Figure 11 shows the result of the mapping of two of these segments. The center line of this figure is also the line that connects the secondary nulls in Figure 4. Here the outer boundary is the secondary null-null line, and ihe secondary nulls have been marked $A^{\prime}$ and $B^{\prime}$. On the right half of the figure the intersection of the sheet of lines, $\Sigma_{A}: x>0$, that converge on the prinary $A$ null is sown. Since this sheet is wound in a scroll, there are an infinity of intersections, of which the first few are illustrated. The cusp on which these curves accumulate is the intersection of the spindle lise, $\gamma_{B 2}$, with this plane. The lines that intersect this plane but do not converge on the primary $A$ null open to the IMF, similar to the lines lying between $\gamma_{A 2}$ and $\gamma_{B 2}$ in Figure 4.

The left side of Figure 11 similarly shows the behavior of the sheet, $\Sigma_{B}: x<0$, 
of lines that emanate from the primary $B$ null. It reflects the overall symmetry of the configuration.

The intersections of both $\Sigma_{A}$ and $\Sigma_{B}$ are symmetric about the vertical axis in Figure 11. In fact, the two scrolls intersect in a complex pattern. Figure 12 shows how this pattern of intersection is mapped to the plane used in Figure 11. The intersection of the two scrolls is represented here as an infinite checkerboard, stretched, distorted, and crowded inside the circle. The undistorted checkerboard is shown in Figure 13. The double lines in the figure correspond to the cusp points, $\gamma_{A 2}$ and $\gamma_{B 2}$ in Figure 11. When these lines are pulled to a point, the lines labeled $A$ and $A^{\prime}$ join, as do the lines $B$ and $B^{\prime}$, etc. Eschert can well represent the style of this configuration. In this figure the separate regions have been labeled by the number of times the lines circle the secondary null-null line.

In the dipole-IMF configuration there are an infinity of topologically distinct flux tubes. These are distinguished by the number of times the flux lines encircle the secondary null-null line. IMF lines do not pass through this ring at all, those that pass through near the coordinate origin are linked once, and so forth. Lines on the boundary between different flux tubes do not join the IMF at both ends, and so are less strongly linked. They are joined to one of the primary nulls instead. At the intersections where four flux tubes come together, the lines both begin and end on nulis. Thus there is an infinity of nuil-null lines in this configuration.

Figure 14 shows how the distant IMF maps into the reference plane. It represents the field lines through a disc of radius 1.5 centered at $(0 .-5,-6)$ and oriented appruxirnately normal to the lines. The labelis on the flux bindles give the number of times the lines eventually encircle the secondary null-null lines. The region labeled with 0 are interplanetary lines and surround the cylinder of those lines encountering the dipolar field. The lines forming the boundary of the cylinder map into the lines shown in Figure 6a, and the lines labeled by the points at $\Sigma_{A 1}, \gamma_{B 2}$ and $\gamma_{B 1}$ are to be identified with those of Figure 4 with similar labels.

A bundle of flux with a circular footprint in the '2' region of our reference plane, Figure 12, is shown in Figure 15. There we see that the bundle does suffer some

† Escher, M. C., "The world of M. C. Escher," e.g. plate No. 239. J. L. Lochner ed., New York: Abradale Press, 1988. 
deformation as it passes through the dipolar field wnle it's irtegrity is preserved. In contrast, we show in Figure 16, a group of four lines that straddle islands '1', '2', and ' 3 ' about the appropriate $X$ point in Figure 12. The numbers to the left and right of the figure identify the lines on either side and represent their origin in the plane of Figure 12. The line labeled 2 ' originates in the lower ' 2 ' island of the plane. Note how these split apart as the null points are passed. This effect was shown even more dramatically in the group of lines shown in Figure 7 that straddle more closely the same $X$ point as the lines of Figure 16 .

\section{COMPARISON WITH A SIMILAR CONFIGURATION}

A recent paper by Birn, et al., (1989), is closely related to this work, though it appears quite different. Their magnetic model represents a plasmoid in the Earth's magnetotail. Further, there are no magnetic nulls in their configuration. Thus the similarities and differences between our results are quite illuminating.

They take a field that can be obtained from the vector potential

$$
\boldsymbol{A}=A_{y} \hat{e}_{y}+A_{x} \hat{e}_{x}
$$

where

$$
\begin{aligned}
& A_{y}=\psi_{c} \tanh \left(\dot{\psi} \psi / \psi_{c}\right)-z^{2} / 2 \\
& A_{z}=-B_{y} x
\end{aligned}
$$

with

$$
\psi=\left(x-x_{3}\right)^{2}\left[2\left(x-x_{1}-x_{2}+x_{3}\right)^{2}+\left(2 x-x_{1}-x_{2}\right)^{2}-3\left(x_{1}-x_{2}\right)^{2}\right] / 24
$$

Various constants are given by

$$
\begin{aligned}
x_{1,2} & =x_{n}-a\left\{1+,-\left[1-(y / b)^{2}\right]^{1 / 2}\right\} \\
x_{3} & =x_{f}-e\left[1-(y / c)^{2}\right]^{1 / 2} \\
c & =b\left\{1-[(d-a) / 3 a]^{2}\right\}^{1 / 2} \\
d & =x_{n}-x_{f} \\
e & =2 a-d / 2
\end{aligned}
$$


and we take

$$
\begin{aligned}
B_{v} & =-0.1, \\
a & =40, b=10, \\
x_{n} & =-20, x_{f}=-100, \\
\lambda & =1 / 40^{3}, \psi_{c}=10 .
\end{aligned}
$$

The square roots are taken to vanish when the arguments are negative.

When $; y l$ is large, field lines lie on the surfaces of constant $A_{y}$ shown in Figure 17a. These surfaces are independent of $y$, in the appropriate range, so here the configuration is essentially two dimensional. We first consider the field line at the center of the $X$-point at $x=-100$. This line is topologically distinct. Specifically, nearly all neighboring lines diverge from it and connect to indefinitely large values of $x$, positive or negative. Thus the line at $x=-100$, and branches of the $X$ that connect to it, are distinguished in that they connect to large $y$, at finite values of $x$. We will show that the $x=-100$ line plays a very similar role to the null points of the dipoleIMF model that wa have considered in the previous sections. In particular, the overall field configuration would be essentially unchanged if the field strength were forced to be slowly decreasing along the length of the $X$-line, until a null appeared at a distant point. 'This illustrates the fact that the three-dimensional configuration around nul! points is frequently the generalization of the two-dimensional configuration around $X$-points (Greene, 1988). The essential feature of a null point from a topological point of view is that there is a sheet of lines that connect to it and divide the space in the neighborhood. These are the surfaces $\Sigma_{A}$ and $\Sigma_{B}$ introduced in Section 3. The distinguished line segments $x=-100$, positive $y$, and $x=-100$, negative $y$ are also at the center of sheets of lines, and so share a significant topological property with null points. These sheets can be constructed as follows. Since $B_{y}$ has been chosen to be negative, lines that lie on branches II and IV of Figure $1 \overline{7}$ a asymptotically approach, in the limit of large positive $y$, the line at $x=-100$. Similarly, lines that lie on branches I and III asymptotically approach, in the limit of large negative $y$, the line $x=-100$. Thus for negative $y$ the line at $x=-100$ has the properties $o^{f}$ an $A$ null and branches I and III form part of a $\Sigma_{A}$ surface, while for positive $y$ this line has the character of a $B$ null. Note that the lines that form branches II and IV for negative $y$, and branches I and III for positive $y$, get caught up the in the 
thre-dimensional field that lies between $y=-10$ and $y=10$, and never asymptote on the line $x=-100$. Thus these branches have no strict topological signińcance. The field depends significantly on $y$ in the region between $y=-10$ and $y=10$. A plot of the surfaces formed by $B_{x}$ and $B_{2}$ at $y=0$ is shown in Figure $17 \mathrm{~b}$.

The field spirals within an "jislard" centered on $x=-100$ at $y=0$. As a result the extension into $y<10$ of the sheet of lines that forms branch II for positive $y$ in Figure $17 \mathrm{a}$ is distorted into a scroll. Similariy, the extension of the lines that form branch I for negative $y$ is wrapped in a different scroll. These two scrolls intersect, and divide space into a number of flux bundles. The curves of intersection are magnetic lines that are asymptotic to the line $x=-100$ for both positive and negative values of $y$. The intersection of these two scrolls forms the complex patterns seen in the paper of Birn et al. (1989). There is another pair of distinguished line segments in this model, tha: lie at the cusp of Figure 17a at $x=-60$. Just as with the line $x=-100$ that lies at the center of the $X$ for large values of $y$, there are sheets of lines that asymptote to the cusp line for large positive and negative values of $y$. The extensions of these sheets mutually intersect in the disturbed region that lies between $y=-10$ and $y=10$. The intersection curve is asymptotic to the cusp line, $x=-60$, for both positive and negative $y$, and crosses the $y=0$ plane at $(-79.60772,0,0)$. This line is shown in bold in Figure 18.

This intersection is a magnetic line that extends to infinity at finite $x$. Thus it is a distinguished line. It serves as a center around which the scrolls, that were discussed in the previous paragraph, wind. In Figure 18 a few of the lines that form one of the scrolls are shown winding around the central cusp-cusp line. There is considerable similarity, with some differences, between this plasmoid model and the dipole model that was discussed in previous sections of this paper. In either case the magnetic field is divided into flux bundles by intersecting scrolls, and these scrolls are formed of lines the asymptotically approach distinguished configurations. In the dipole case these distinguished configurations are the primary null points in the notation introduced at the end of Section 2, and in the plasmoid case they are the lines at $x=-100$ that run to infinite $y$ at finite $x$. Further, the scroll is wound around a line that connects secondary nulls in the notation of Section 2 in the dipole case, and around the line that connects the distinguished line segments at $x=-60$ 
in the plasmoid case. Thus the essential identification between the the two models is between null points and lines that are bounded in the $x$-direction. In summary, the field is split into a number of distinct flux bundles that differ in the number of loops that they make around the cusp-cusp line. These flux bundle: are separated by sheets of lines that converge on either of the $X$-lines.

Another configuration exhibiting intersecting scrolls has been given by Lau and Finn (1991). It was also based on plasmoid formations. There the scroll from one side is manifested by following an almost continuous sheet of lines originating form their model's counterparts of branches II and IV of our Figure 172. A continuous folding is sem as the intersections of this sheet of lines in various $y=$ constant planes are followed from large positive $y$ to large negative $y$. Since this sort of pattern has been shown to exist in at least thres quite distinct configurations, there is reason to expect that it might occur fairly commonly.

\section{CONCLUSIONS}

Here we have inalyzed a complex field configuration, consisting of the dipolar field of a distribut, 1 ring current immersed in a constant IMF.

In the absence of an IMF, the field lines produced by the ring current form closed loops that encircle a closed curve along which the magnetic field strength vanishes. The latter curve we will call the null circle. When this configuration is perturbed by superimposing a small constant field, the null circle acquires a tangential field component, except at two points that retain the null property. In this paper, these two nulls were called the secondary nulls. Thus the null circle becomes a a pair of lines of force that join a pair of nulls. We call such lines null-null lines. Thus null-null lines of force take on the properties of curves of vanishing field strength, but they have a tangential magnetic field component so that they are locally undistinguished. On the other hand, they retain the character of being distinctive among all the field lines in that they end on null points, and thus form part of a closed curve.

Under the perturbation, the closed loops encircling the ring current are transformed into helical lines of force. A null-null line lies at the center of these helices. Since the null-null lipes form a closed loop, the number of times a helix threads this loop can be determined unambiguously. Most lines make a finite number of loops 
before joining the IMF, and for every number, there is some flux bundle of lines with that many loops.

The number of loops made by a line of force is an integer, not a contintious variable. Therefore there is singular behavior at the borders between flux bundles where the threading number is not coutinuous. We have shown here that these borders are formed of lines of force that join to another set of null points, which were called the primary nulls.

Thus null points, and the lines that join to them, form the skeleton of a structure containing an infinite number of topologically distinct flux bundles. In short, we have given another example where such a skeleton is essential in establishing the topology of the magnetic field lines.

\section{References}

Birs, J., Hesse, M. and Schindler, K., "Filamentary structure of a three-dimensional plasmoid," $J$. Geophys. Res. 94, 241-251 (1989).

Cowley, S. W. H., "A qualitative study of the reconnection between the earth's magnetic field and an interplanetary field of arbitrary orientation," Radio Sci. 8, 903-913 (1973).

Fulkao, S., Ugai, M. and Tsuda, T., "Topological study of magnetic field near a neutral point," Rep. Jonos. Res. Jpn. 29, 133-139 (1975).

Greene, J. M., "Geomatrical properties of three-dimensional reconnecting magnetic fields with nulls." J. Geophys. Res. 93, 8583-85;0 (1988).

Lau, Y.-T. and Fina, J. M., "Three-dimensional kinematic reconnection of plasmoids," Astrophys. J., 366, 577-591, (1991).

Ste:n, D. P., "The role of $\mathrm{O}$-type neutral lines in magnetic merging during substorms and solas flares, ${ }^{n}$ J. Geophys. Res. 84, 63-71 (1979).

Stern, D. P., "A study of the electric field in an open magnetospheric model," J. Geophys. Res. 78. 7292-7305 (1973).

\section{Ackizouledgments}

One of the authors (M.S. Chance) would like to express his appreciation for the hospitality cffered to him during his sabbatical leave in 1987-88 by his colleagues at General Atomics where a substantial portion of this work was carried out. We would also like to thank Peter Willmann of Lawrence Livermore National Laboratory for his assistance in using the GRAFL3 package upon which the graphics in this paper were constructed. 


\section{APPENDIX A: COALESCENCE CURVES}

In Section 2 it was noted that the pair of nulls in a given quadrant can coalesce along the locus where the two sets of level curves in Figure 2 for the dipole field osculate. Here we derive and solve the equation for the locus. It will be seen that the primary nulls always have real ejgensolutions and also that the necessary $X$-point separating the two classes of nulls lies on this curve. The equation for this locus, shown as the dashed line in Figure 2, can be calculated by equating the slopis of the level curves as follows:

Since

$$
B^{2}=B_{p}^{2}+B_{z}^{2}=\text { constant }
$$

and

$$
\tan \omega \equiv \frac{B_{2}}{B_{\rho}}=\text { constant }
$$

for these curves, implicit differentiation and solving for the slopes give

$$
-\frac{B_{\rho} \frac{\partial B_{\rho}}{\partial \rho}+B_{z} \frac{\partial B_{z}}{\partial \rho}}{B_{\rho} \frac{\partial B_{f}}{\partial z}+B_{z} \frac{\partial B_{z}}{\partial z}}=\frac{d z}{d \rho}=-\frac{B_{\rho} \frac{\partial B_{z}}{\partial \rho}-B_{z} \frac{\partial B_{\rho}}{\partial \rho}}{B_{\rho} \frac{\partial D_{z}}{\partial z}-B_{z} \frac{\partial B_{\rho}}{\partial z}}
$$

and, after collecting terms, we get the condition that

$$
\tilde{D} \equiv \frac{\partial B_{\rho}}{\partial \rho} \frac{\partial B_{z}}{\partial z}-\frac{\partial B_{z}}{\partial \rho} \frac{\partial B_{\rho}}{\partial z}=0 .
$$

Since $\tilde{D}$ is proportional to the determinant, $D$, of the $\widetilde{\nabla B}$ matrix for the dipolar field, this vanishing is not surprising in view of the role that this matrix plays in describing the behavior of the magnetic field in the vicinity of a null. This was discussed in Section 3. As noted in that section, this line separates the primary nulls from the secondary ones. Also, by comparing (15) with (A4), it is seen that primary nulls always have real eigensolutions.

For the specific madel discussed in Section 2 (A4) gives essentially a bicubic equation for $z$ :

$$
-z R^{2}\left[-z^{2}\left(R^{2}-5 \rho^{2}\right)\left(2 R^{2}-5 \rho^{2}\right)+\rho^{2}\left(R^{2}-5 z^{2}\right)\left(4 R^{2}-5 p^{2}\right)\right]=0
$$

and the relevant non trivial solution to this cas be shewn to be

$$
z^{2}=\frac{-\left(3 \rho^{2}+2 a^{2}\right)+\left(\rho^{4}+44 \rho^{2} a^{2}+4 a^{4}\right)^{1 / 2}}{4} .
$$


In particular, some solutions for the parameters given in Section 3 are $(\rho, z)=$ $(0,0),(2 a, 0),\left(a, a / 2^{1 / 2}\right)$ and $\left(a / 3^{1 / 2}, a / 3^{1 / 2}\right)$. The root at $(2 a, 0)$ is at a saddle point in the magnitude of $B$, and can be seen in Figure 2. This point migrates along the fixed osculating locus as the IMF and the associated nulls are introduced and varied. This can be shown as follows:

Writing the total field as $B_{t}=B+b$, the saddle point satisfies

$$
\nabla B_{i}^{2}=0
$$

and the $\rho$ and $z$ components give the set of equations

$$
\left(\begin{array}{cc}
\frac{\partial B_{y}}{\partial \rho} & \frac{\partial B_{z}}{\partial \rho} \\
\frac{\partial B_{y}}{\partial z} & \frac{\partial B_{z}}{\partial z}
\end{array}\right)\left(\begin{array}{c}
b_{y} \\
b_{z}
\end{array}\right)=-\frac{1}{2}\left(\begin{array}{c}
\frac{\partial B^{2}}{\partial \rho} \\
\frac{\partial B^{2}}{\partial z}
\end{array}\right),
$$

where $b_{y}$ and $b_{z}$ are respectively the $y$ and $z$ component of the constant IMF. Taking the indicated derivatives on $B^{2}$ and solving for $b$ in the $\rho-z$ plane we find the condition that

$$
\left(\frac{\partial B_{\rho}}{\partial \rho} \frac{\partial B_{z}}{\partial z}-\frac{\partial B_{z}}{\partial \rho} \frac{\partial B_{\rho}}{\partial z}\right) B_{t}=\bar{D} B_{t}=0
$$

Thus (A7) is satisfied at a null $\left(B_{t}=0\right)$, but if the field is finite then $\nabla B_{t}^{2}$ vanishes on the curve given by $\tilde{D}=0$, at the point where either of (A8) is also satisfied. Figure 19 is an example showing the coalescence curve and the saddle point of the total magnetic field with the null at $(0,1,2)$. For clarity, the contours are equally spaced along the straight line joining the origin and the null at $(0,1,2)$.

\section{APPENDIX B: NULL-NULL CIRCLES}

We find here under what special circumstances that there exist circular magnetic field lines which connect to null pairs. We first assume that the magnetic field has azimuthal symmetry and then determine under what conditions that a field line lies along the intersection of a sphere, $r=$ constant, and a plane given by

$$
\zeta \equiv z-\alpha y=0
$$

It proves convenient to use spherical coordinates here. Thus we first require that

$$
\boldsymbol{r} \cdot B_{\mathrm{t}}=0
$$


or

$$
\frac{1}{\sin \theta} \frac{\partial}{\partial \theta}\left(A_{\phi} \sin \theta\right)+r\left(b_{\nu} \sin \theta \sin \phi+b_{z} \cos \theta\right)=0
$$

Using

$$
\zeta=r(\cos \theta-\alpha \sin \theta \sin \phi)=0,
$$

this becomes,

$$
\frac{\partial}{\partial \theta}\left(A_{\phi} \sin \theta\right)+r \frac{\left(b_{y}+\alpha b_{x}\right)}{\alpha} \sin \theta \cos \theta=0 .
$$

The other tequirement takes the form

$$
\nabla \zeta \cdot B_{\imath}=0
$$

or,

$$
\frac{\partial}{\partial r}\left(r A_{\phi} \sin \theta\right)+r\left(b_{z}-\alpha b_{y}\right) \sin ^{2} \theta=0,
$$

where we again used ( $B 4)$.

These assumptions are fulfilled for the class of vector potential $A_{\phi}$ which has the form

$$
A_{-\phi}=H(r) \sin \theta
$$

where $H(r)$ is a function of $r$ only. Then the angle $\theta$ drops out of $(B 5)$ and $(B 7)$ and these two equations can be solved for $\alpha$ and the particular value of the radius $r$ of the circular feid iines. Our model as well as the point dipole satisfy this condition. That :herc actually are nulls on these circles at $x=0$ follows from the observation in (3) that $B_{x}=0$ there for our configuration.

It is straigintforward tc evaluate the tangential componert of the field along the null-null circle. Thus

$$
\begin{aligned}
\frac{B \cdot(\nabla \zeta \times \nabla r)}{\left(1+\alpha^{2}\right)^{1 / 2}} & =\left[-\alpha B_{\theta}+\left(b_{y}+\alpha b_{z}\right) \sin \theta\right] \frac{\cos \phi}{\left(1+\alpha^{2}\right)^{1 / 2}} \\
& =b_{y}\left(i+\alpha^{2}\right)^{1 / 2} \sin \theta \cos \phi
\end{aligned}
$$

where we used (B7). It can also be seen here that the magnitude of $B$ vanishes where $\cos \phi=0$, so that there are magnetic rulls at those points on the circle that has been caiculated here. Further, the tangential field has opposite sign along the two semicircles that connect the nulls. Thus the line of force does not go around the circle and close on itself, but is composed of two semicircles on which the field is oppositely directed. 


\section{Figure Captions}

Figure 1 Contours of the magnetic dipole flux, $\psi$, for the magnetic field of the distributed ring current model.

Figure 2 Level curves of the orientation angle, $\omega$, and the magnitude of the dipolar field. $|B|$, for the magnetic field in the first quadrant of Figure 1. The contour lines of $|\boldsymbol{B}|$ are equally spaced along the diagonal of the figure.

Figure 3 The principal magnetic field lines in the vicinity of a null point for an eigensystem with real (upper figures) and complex (lower figures) eigenvalyes.

Figure 4 The magnetic field line configuration in the $x=0$ plane. The principal field lines in this plane which intersect the primary nulls at $A$ and $B$ and spira] into the secondary nulls are the heavy lines.

Figure 5 A three-dimensional stereo view of the principal field lines which passes through the nulls. The field line comprising the null-null circle connecting the secondary (complex) nulls is not shown here.

Figure 6 The field lines along the $\Sigma_{A 1},(\mathrm{a})$, and the $\Sigma_{A 2}$, (b), surfaces about the primary $A$ rull.

Figure 7 Three field lines in the neighborhood of the one in $\Sigma_{A 2}$ which encircles the secoudary null-null circle once and connects to the primary $B$ null.

Figure 8 Magnetic fieid lines originating from a small circle displaced somewhat away form the null along $\gamma_{A 1}$ (refer to Figure 4). These are distinguished by those lines near to $\Sigma_{A 1},(\mathrm{a})$, and those near to $\Sigma_{A 2},(\mathrm{~b})$.

Figure 9 Magnetic field lines criginating from a small circle displaced somewhat away from the null along $\gamma_{A 2}$ (refer to Figure 4). These are distinguished by those lines near to $\Sigma_{A 1},(a)$, and those near to $\Sigma_{A^{2}}$, (b).

Figure 10 A mapping of the field lines onto the secondary null-null plane on a coarse grid. The numbers depict where, and the number of times, the field lines intersect the plane.

Figure 11 The mapping of the $\Sigma_{A}: x>0$ (right half of the figure) sheet of lines, and the $\Sigma_{B}: x<0$ (left half of the higure) sheet of lines onto the secondary null-null plane.

Figure 12 Like Figure 11 but including the lines from both positive and negative $x$. The numbers between the intersections depict the number of times those lines not lying on the $\Sigma$ surfaces incersect the secondary null-null plane. This represents Figure 10.

Figure 13 An undistorted but topologically accurate representation of Figure 12. 
Figure 14 A mapping of a portion of the distant IMF onto the secondary null-null plane.

Figure 15 A bundle of Aux wtich passes through the '2' regions of Figure 12.

Figure 16 A group of four lines straggling islands ' 1 ', '2', ' 3 ' of Figure 11. The line labeled 2 ' intersects the lower ' 2 ' island in Figure 12.

Figure 17 Contours of $A_{y}$ in the magnetotail model for large values of $|y|$, (a), and for $y=0,(b)$.

Figure 18 Some selected lines of force in the plasmoid model. The heavy line connects from one cusp segment to the other. One end of each of the light lines lies along branch II $(-85 \leq x \leq-67, y=10)$ in Figure 17a. The labels on the $(x, y, z)$ axes are the true values multiplied by $(1,8,16)$.

Figure 19 Contours of the magnitude of the total magnetic field of the dipole-IMF system. The locus of both the osculating curve of the nulls and the saddle point of $\left|B_{4}\right|$ is shown as the dashed line. 


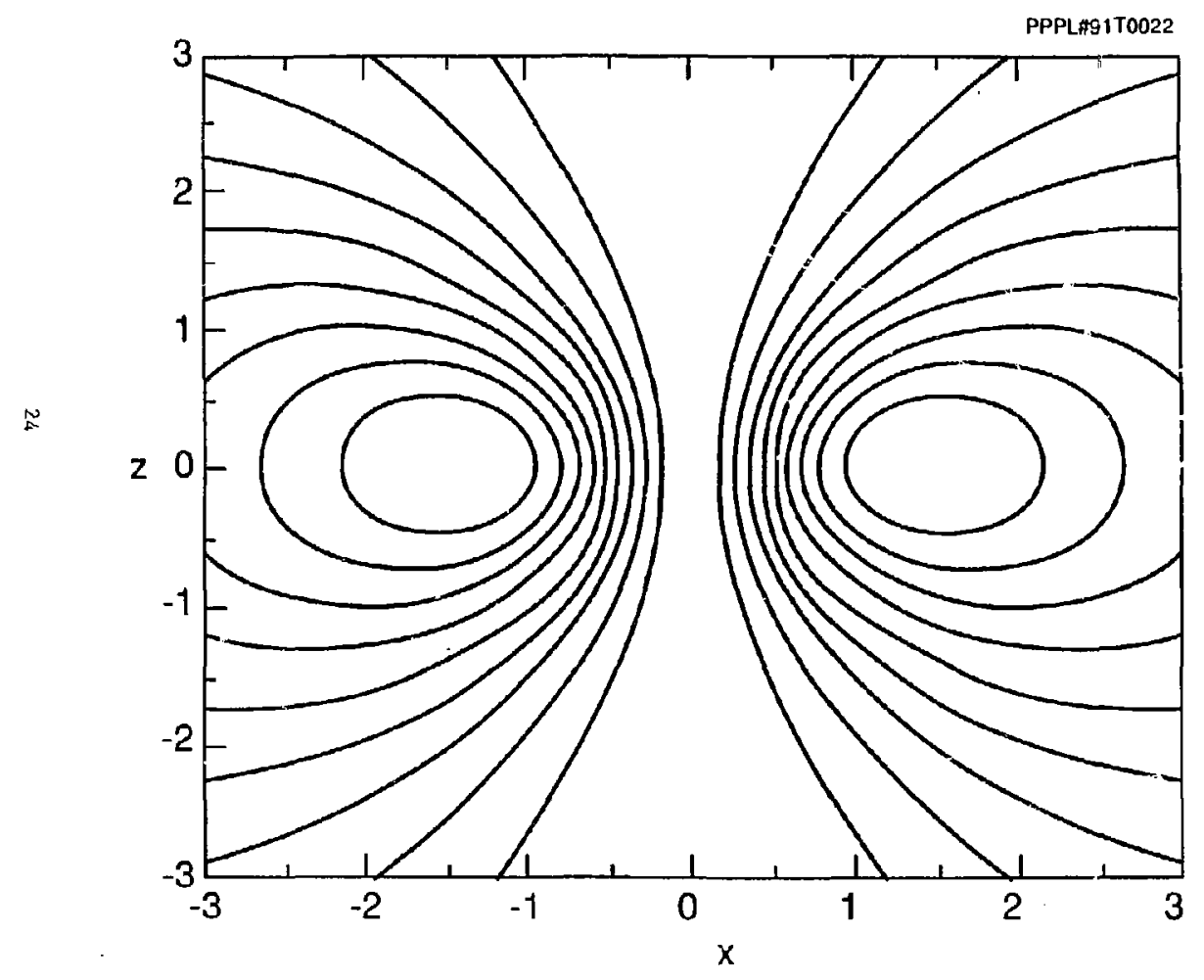

Fig. 1 
$-$

PPPL\#91T0023

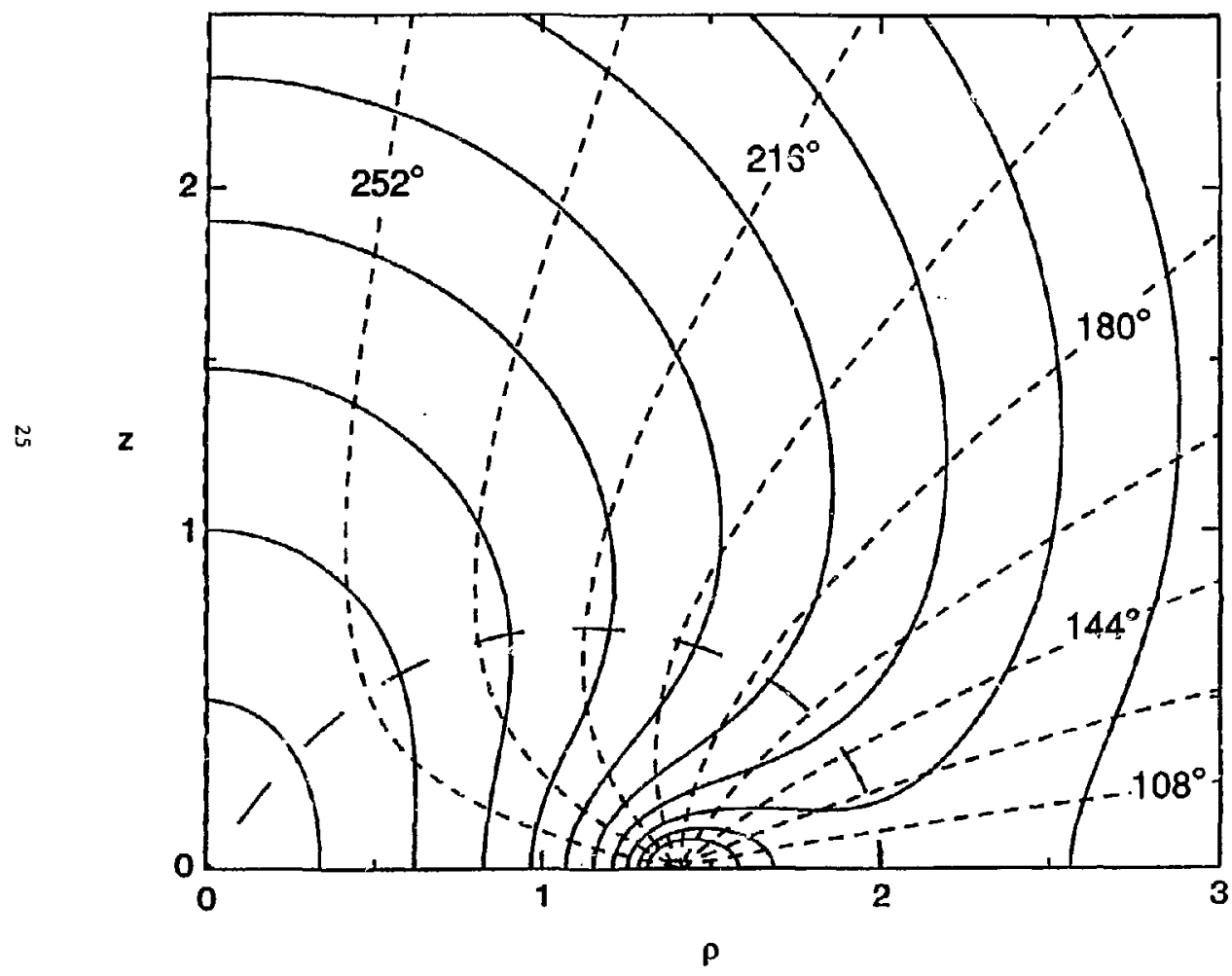

Fig. 2 
แล9T0041

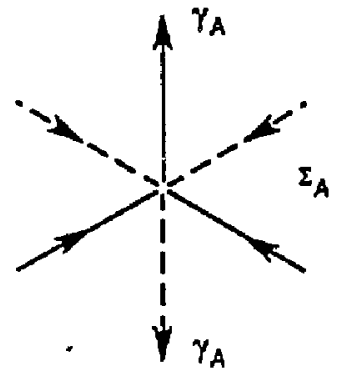

TYPE - A NULL

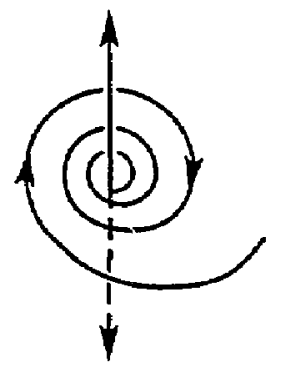

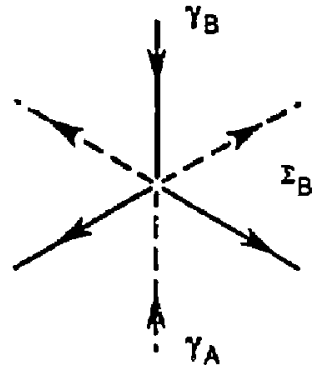

TYPE - B NULL

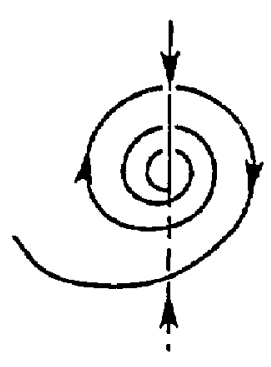

F1g. 3 


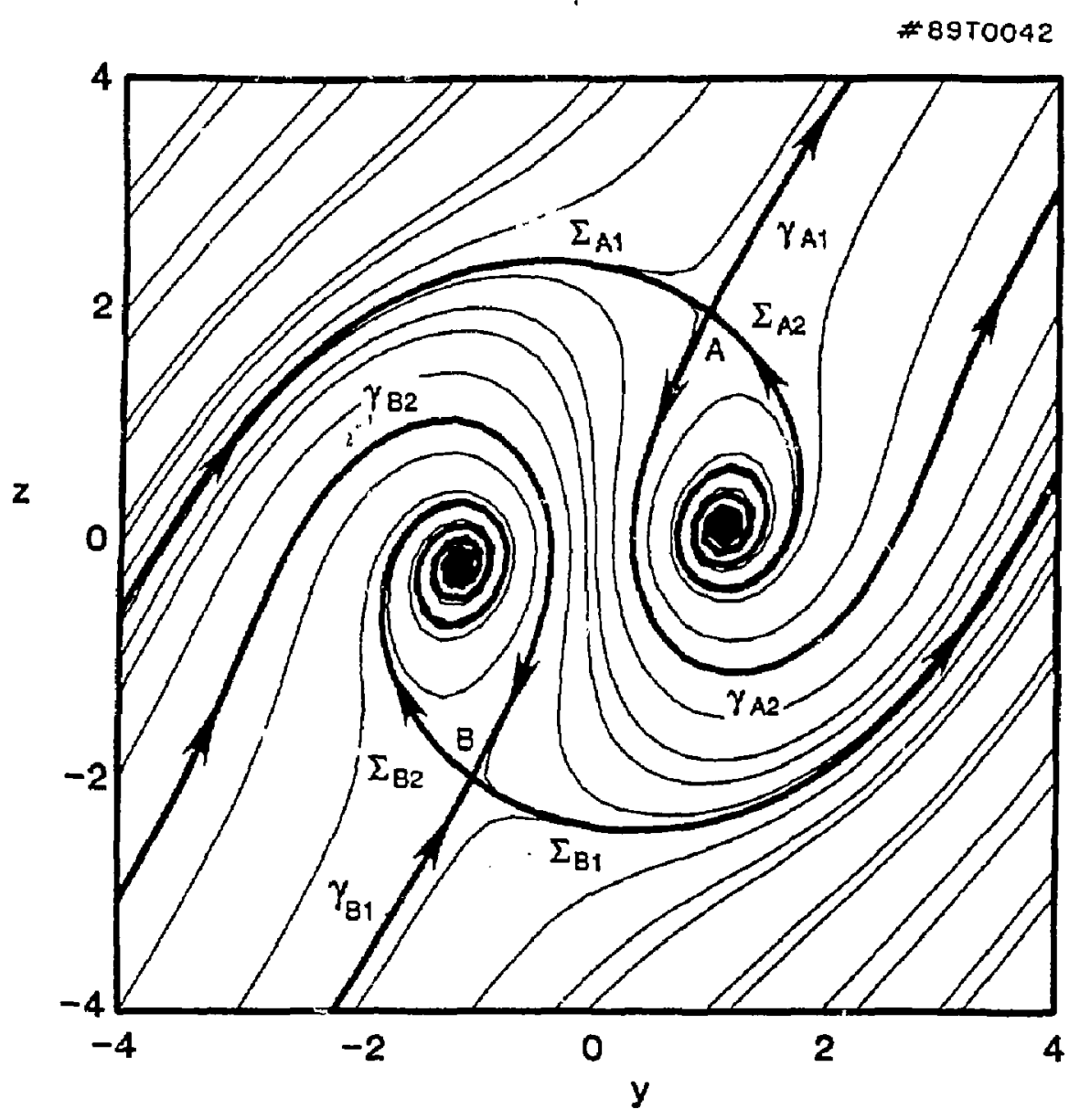

Fig. 4 
OITtO10

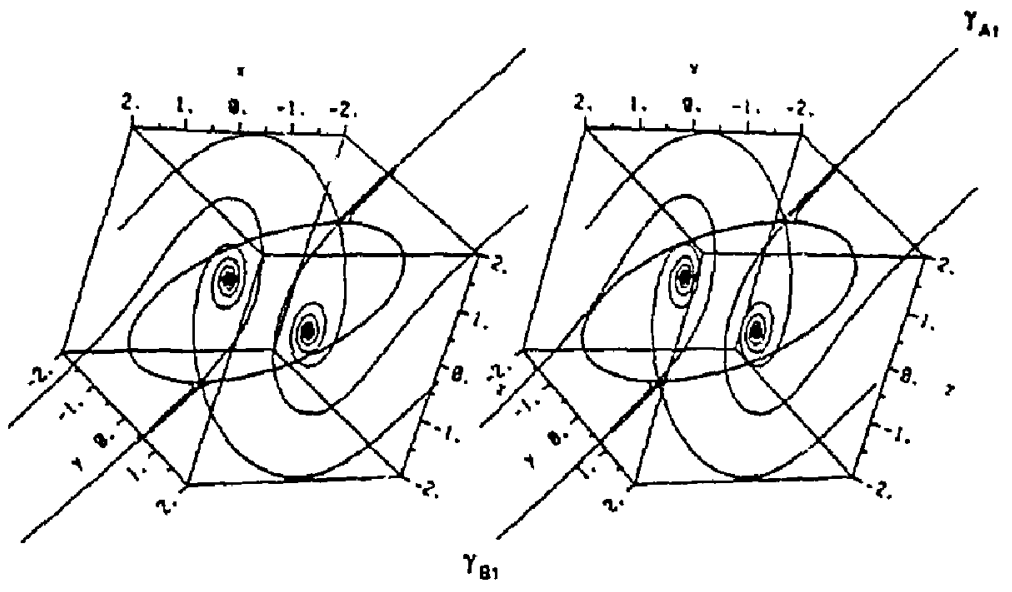

FIg. 5 
9171022

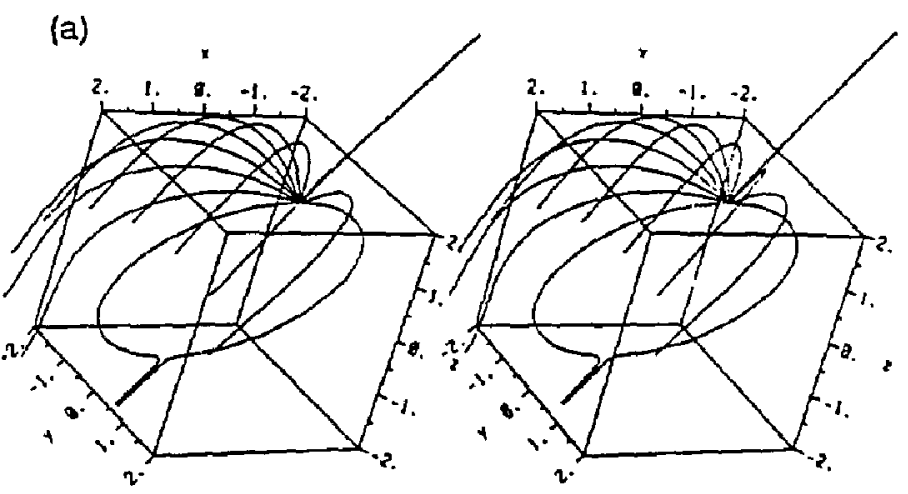

(b)

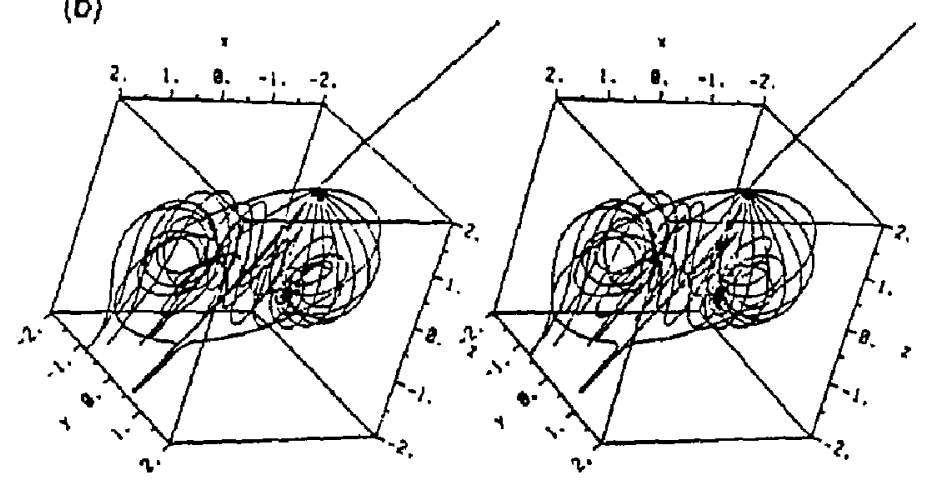

Fig. 6 
$01 T 1017$

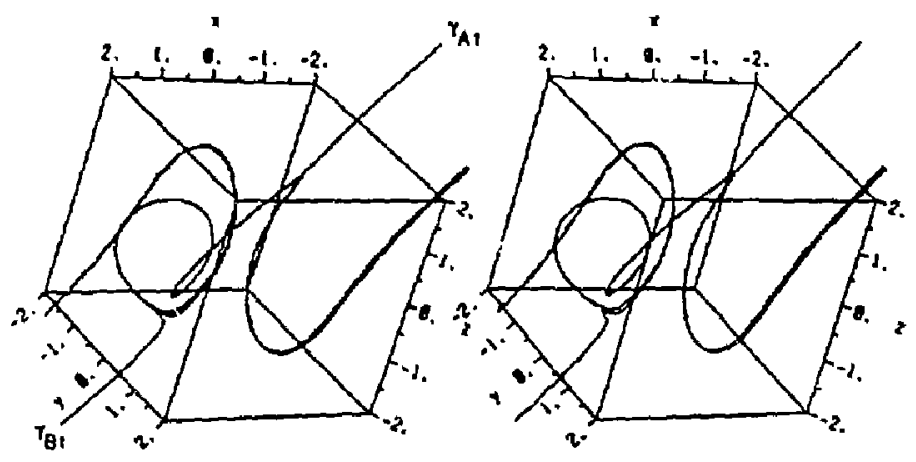

Fig. 7 
9171023
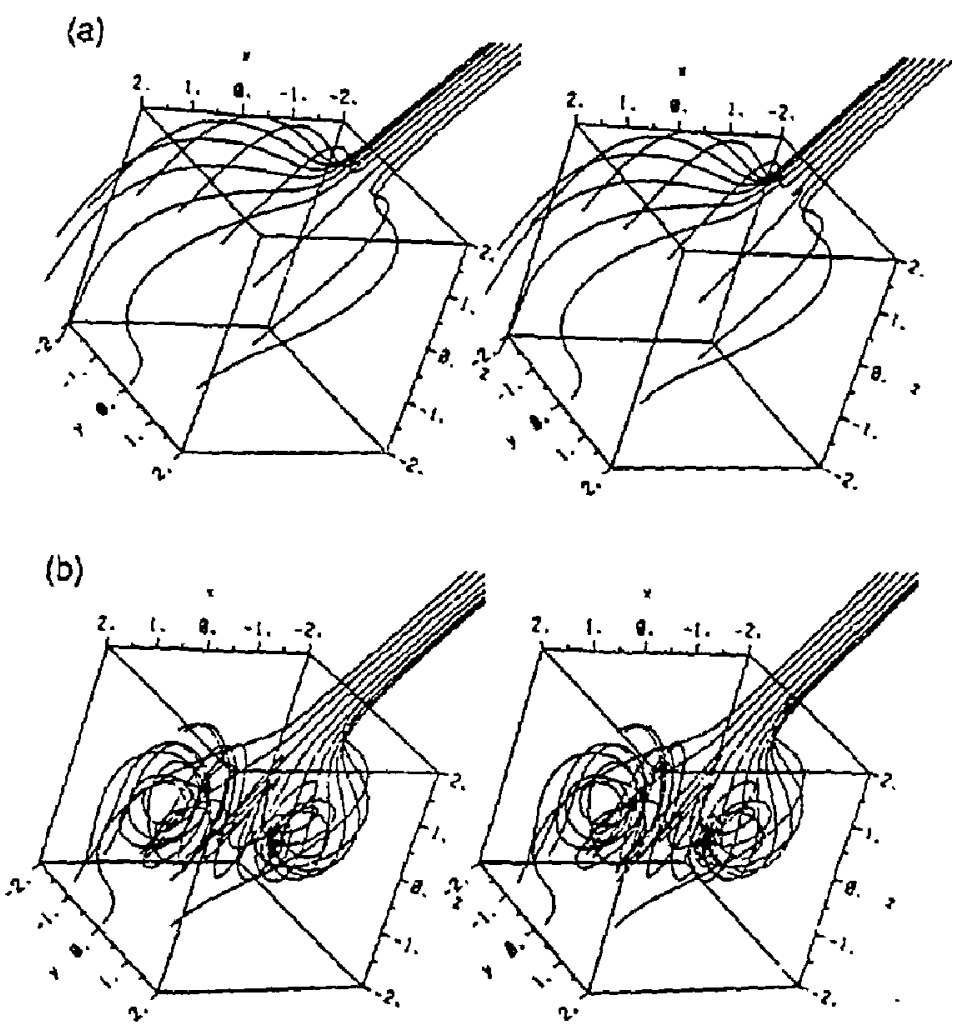

Fig. S 
(a)

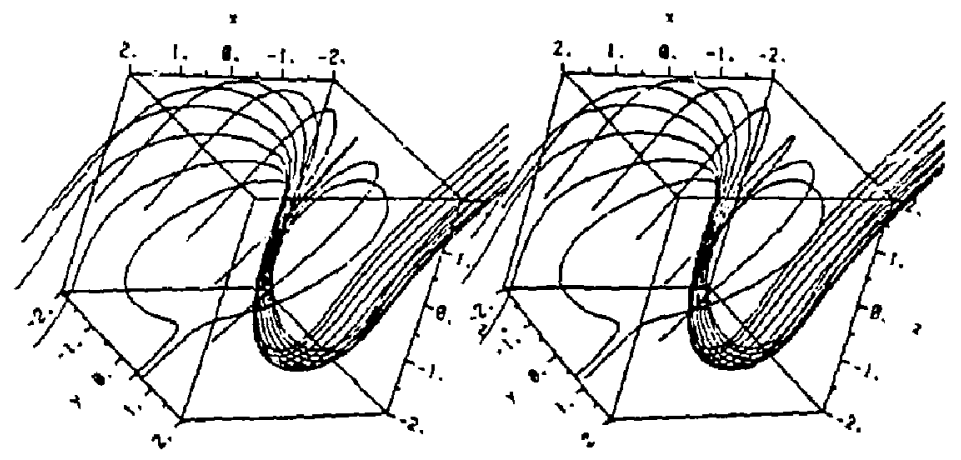

(b)

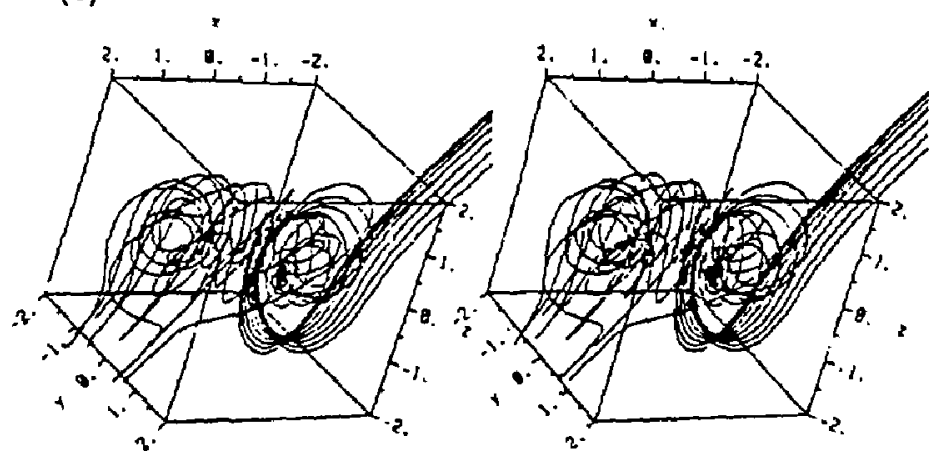

Fig. 9 


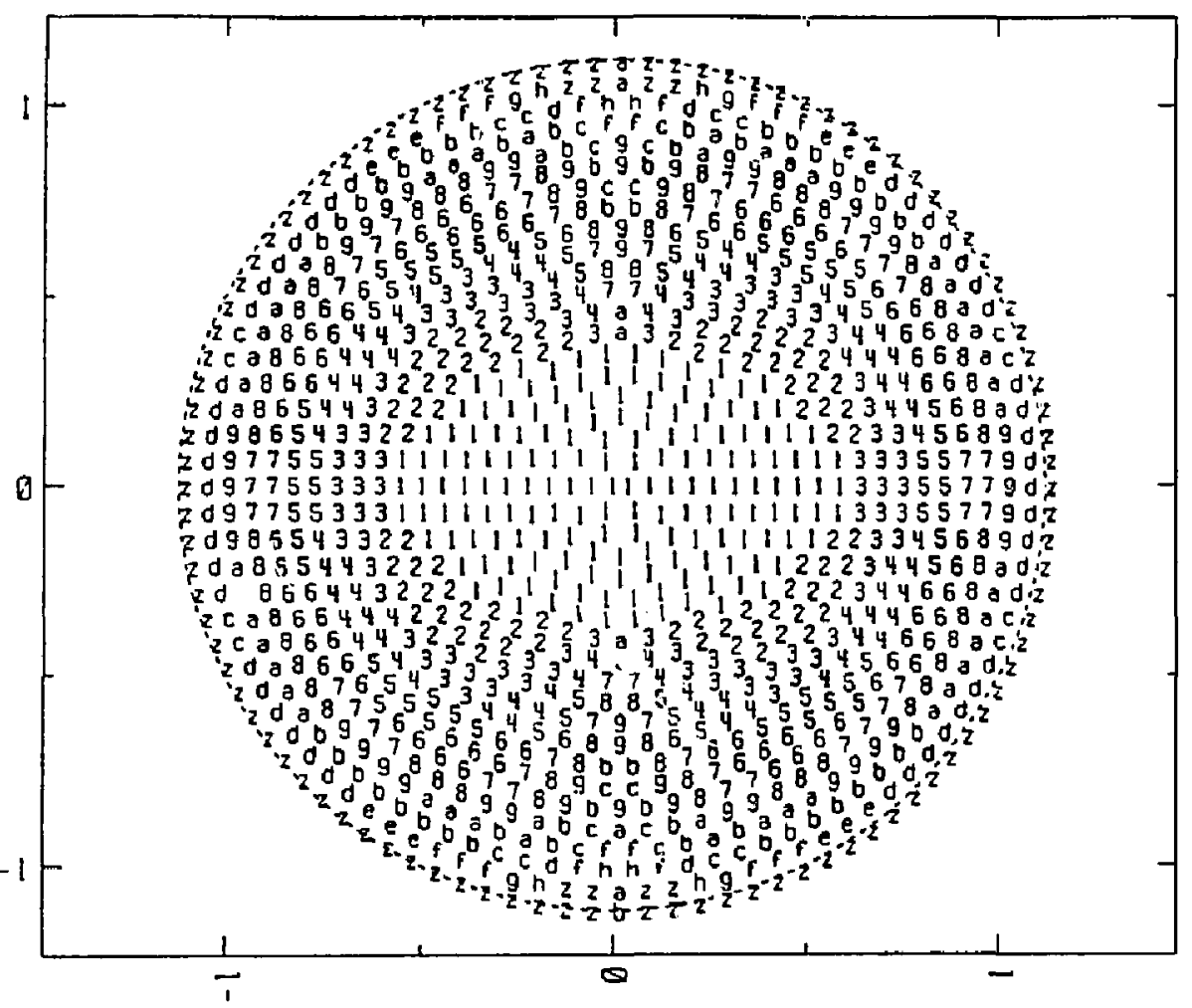


$91 \mathrm{~T} 1013$

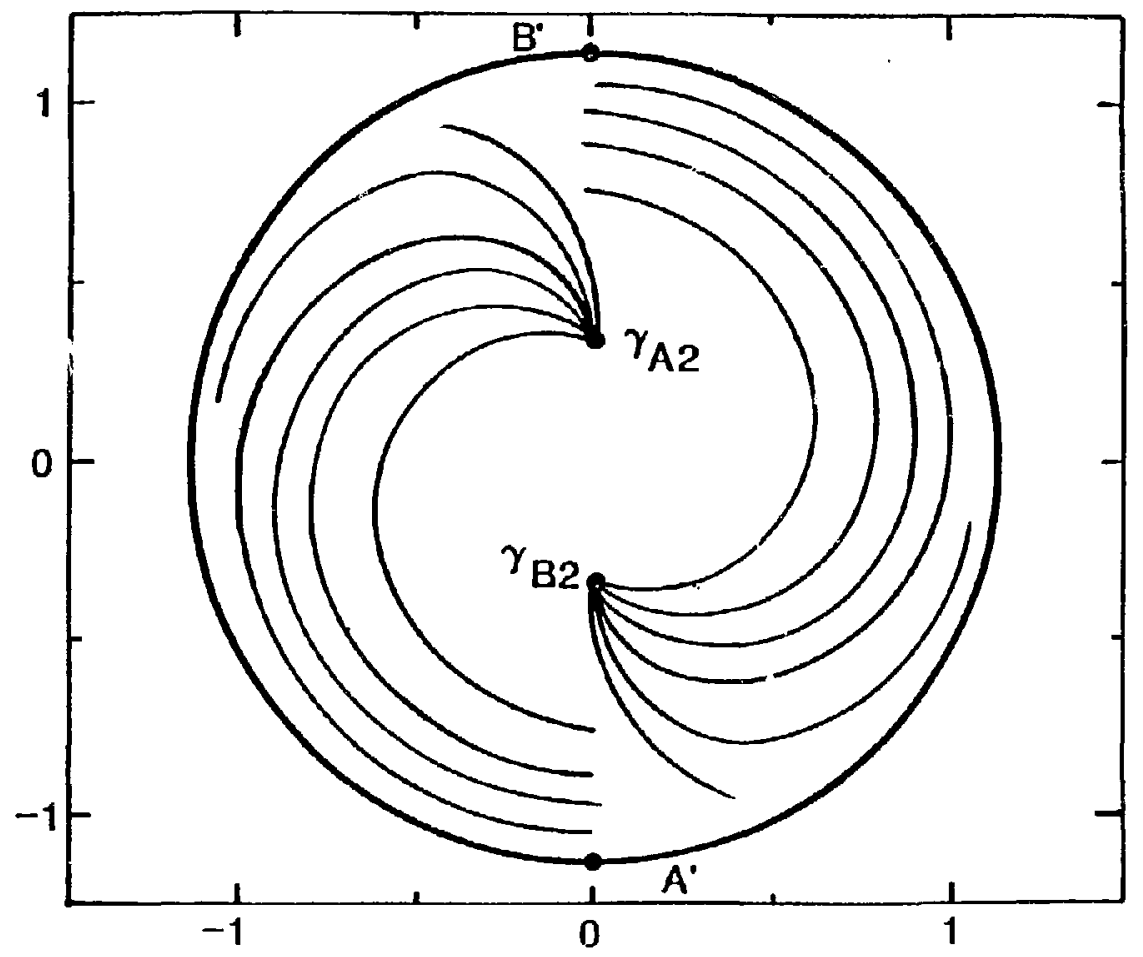

Fig. 11 
$91 \mathrm{~T} 1012$

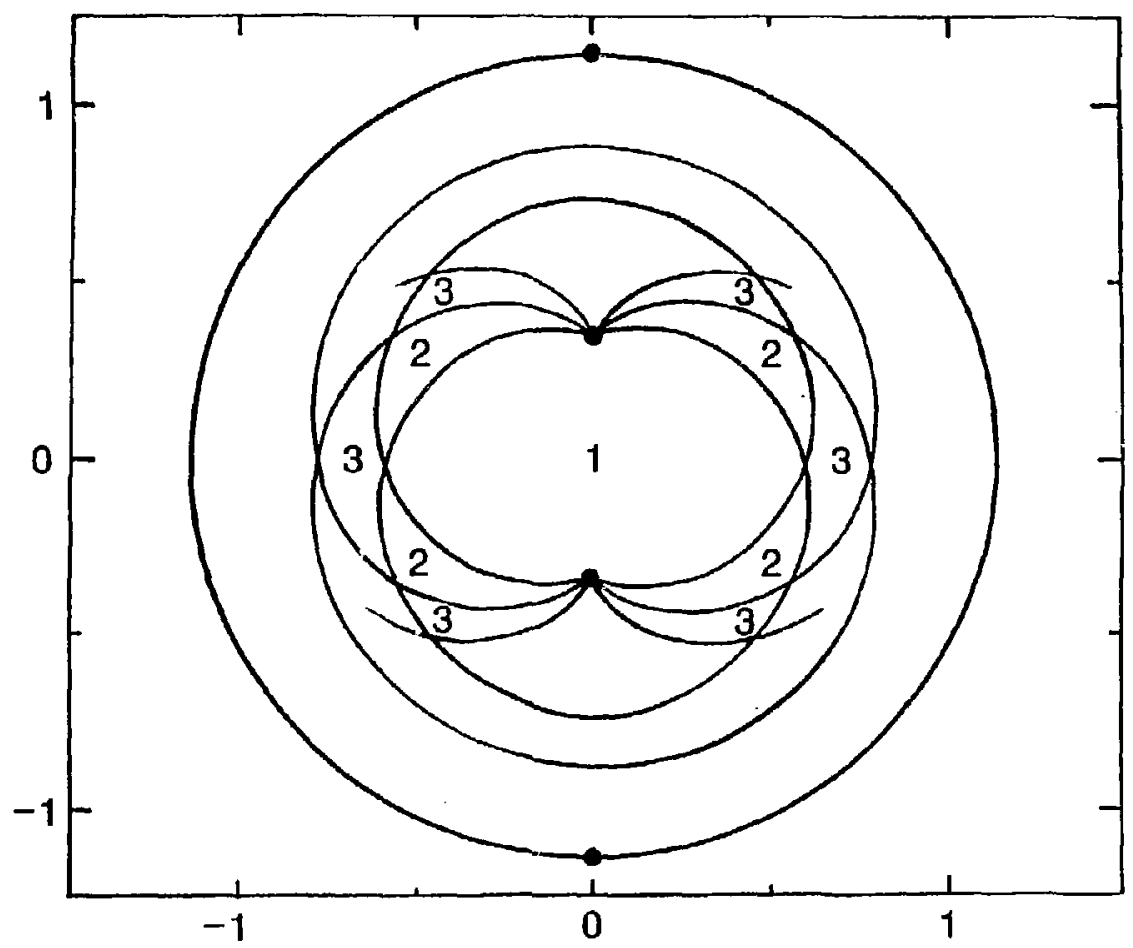

F1g. 12 
$91 \mathrm{~T} 1015$

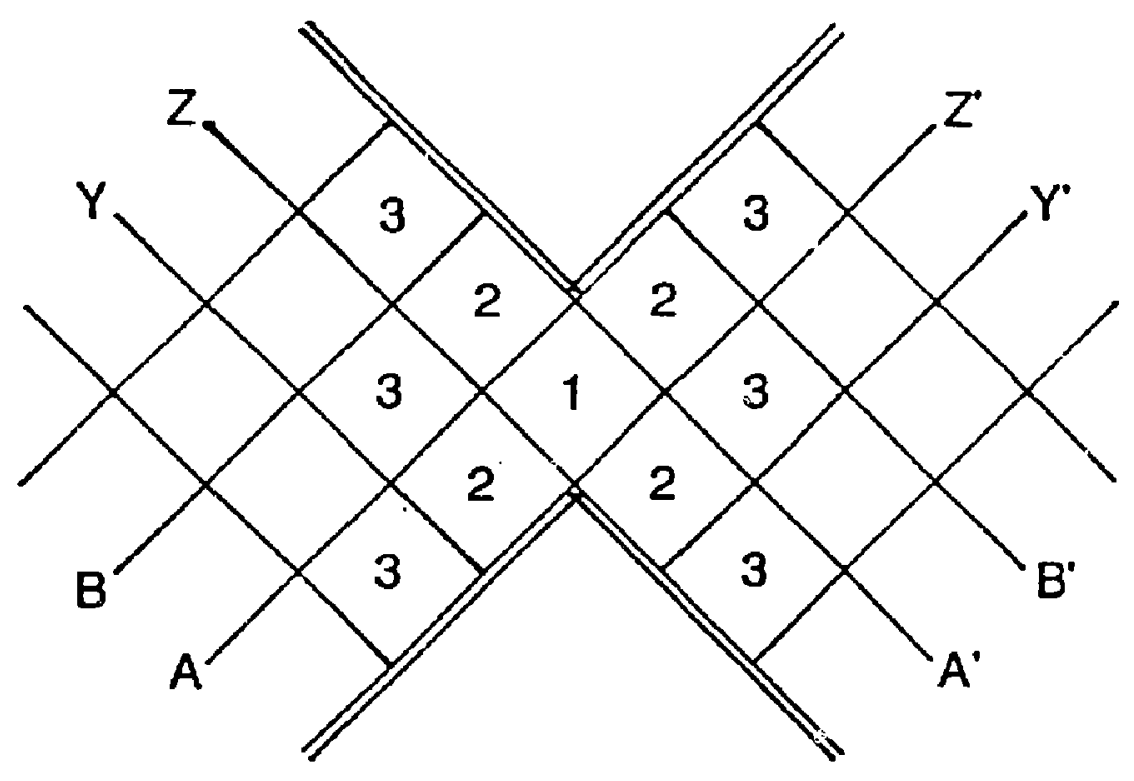


$91 \mathrm{~T} \cup 14$

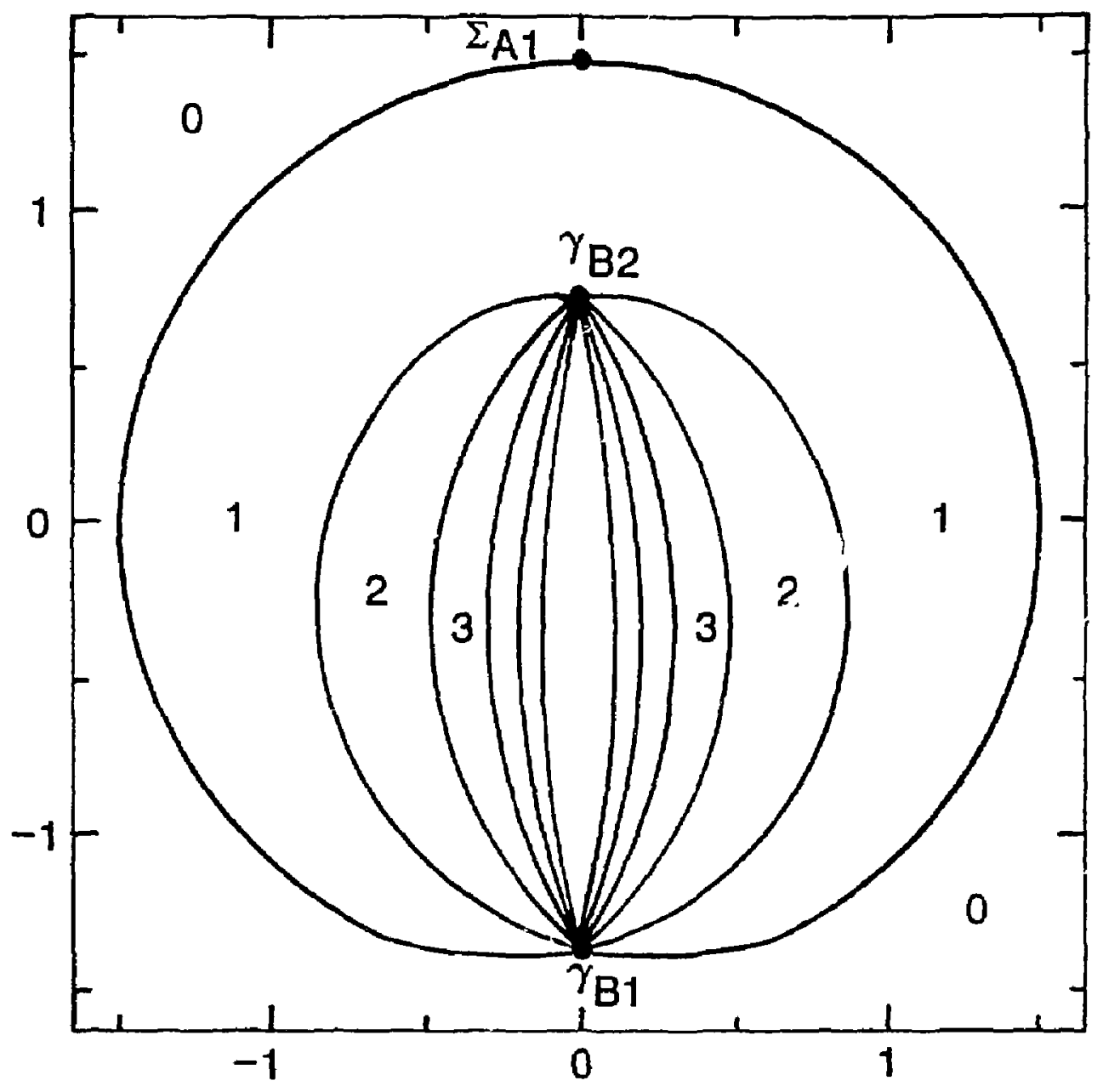

F士g. 14 
g1t1018

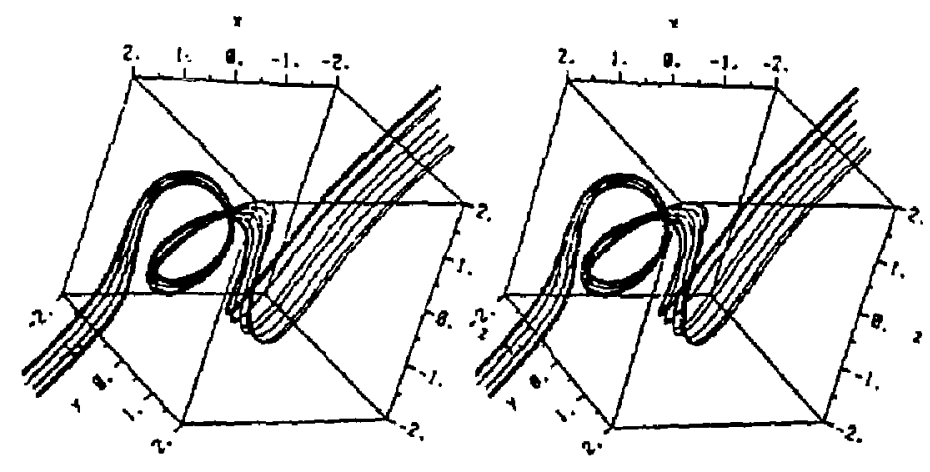

Fig. 15 
Q1T1020

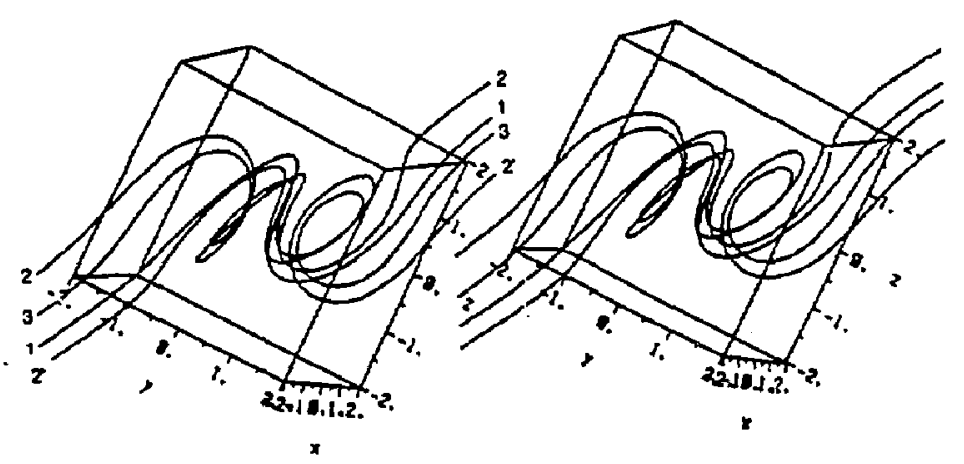

FIg. 16 
0171025

(a)

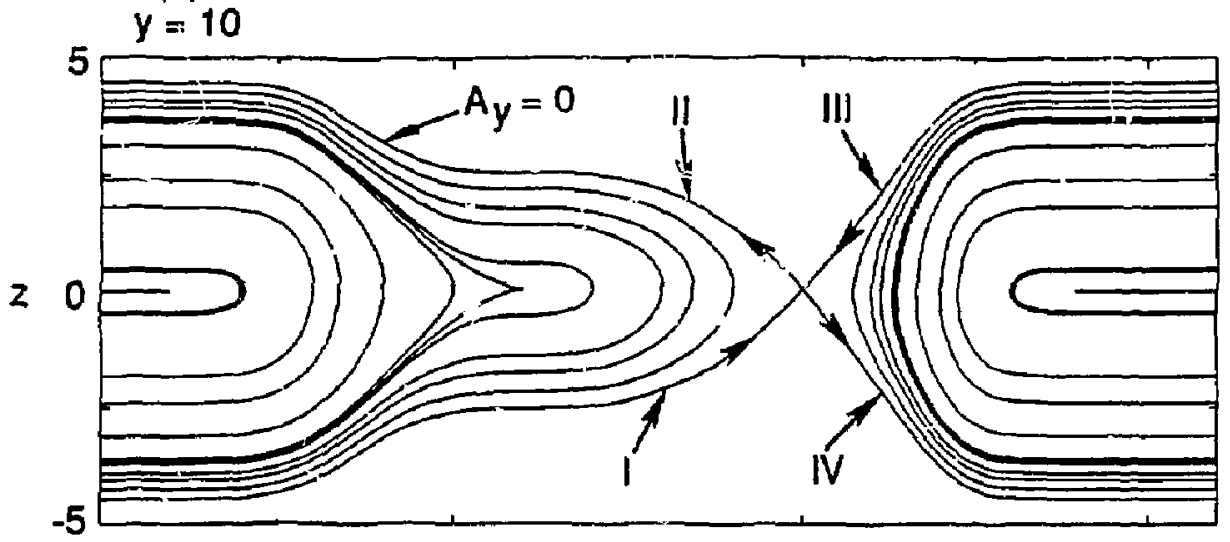

(b)

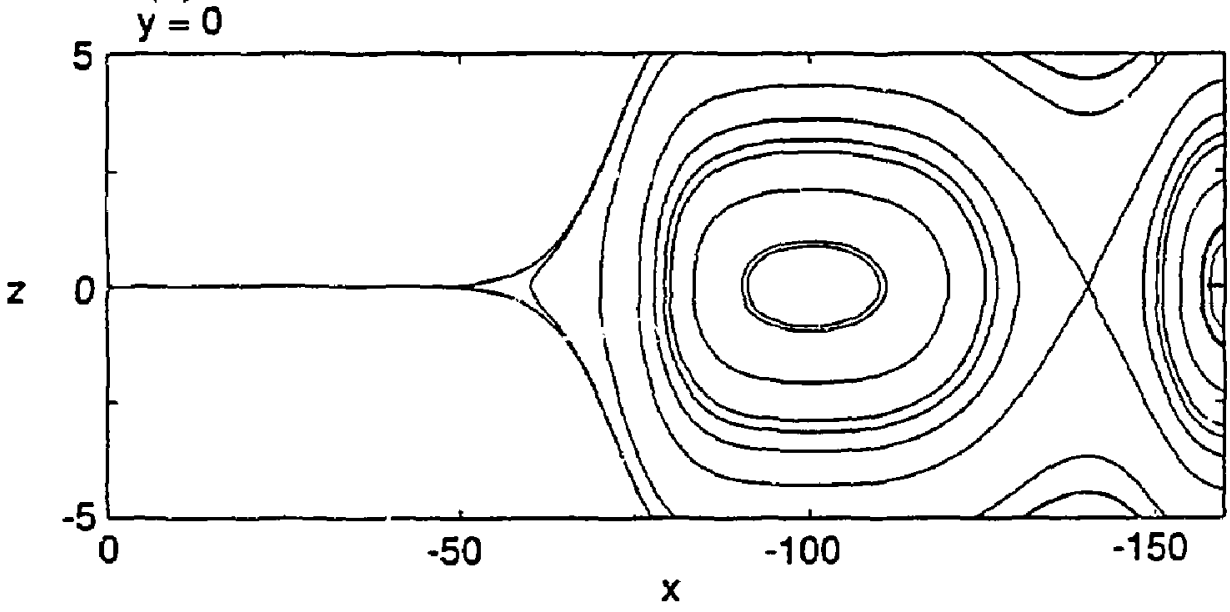

F1g. 17 
9111019

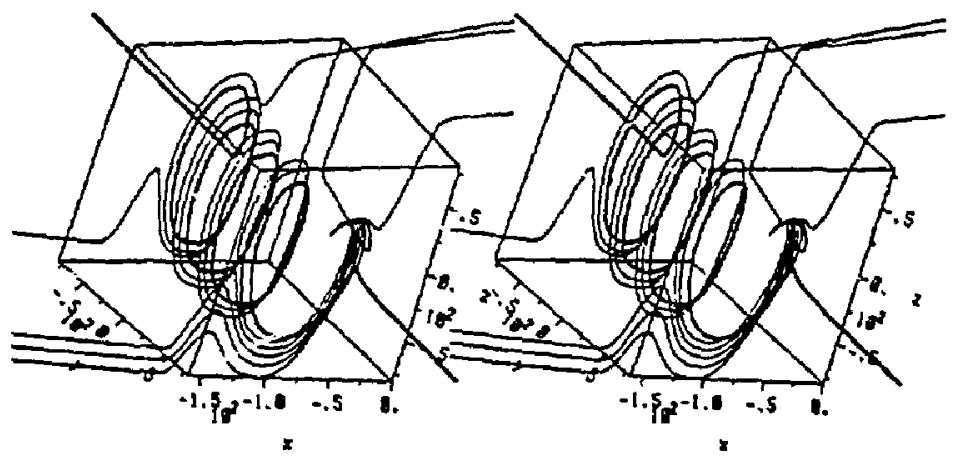

FIg. 18 
\# ө9т0048

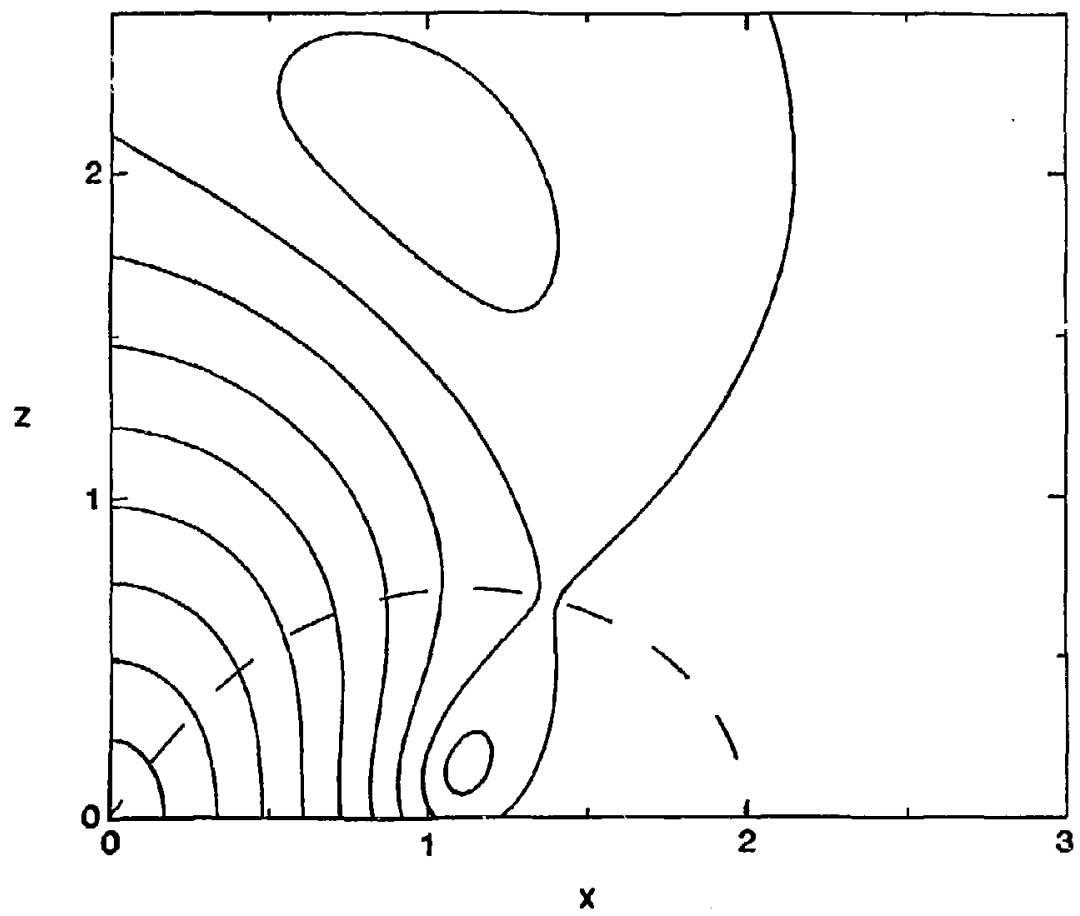

F1g. 19 